Rev. Int. Contam. Ambie. 32 (3) 281-291, 2016

DOI: 10.20937/RICA.2016.32.03.03

\title{
EVALUACIÓN HIDRÁULICA DE UN REACTOR ANAEROBIO DE FLUJO ASCENDENTE (RAFA) USANDO UN MODELO DE DISPERSIÓN AXIAL
}

\author{
Jhonny Isaac PÉREZ MONTIEL ${ }^{1 *}$, Gerardo José ALDANA VILLASMIL ${ }^{2}$ y \\ Roberto Eliecer ROJANO ALVARADO ${ }^{1}$
}

${ }^{1}$ Grupo de Investigación en Sistemas Ambientales, Facultad de Ingeniería, Universidad de La Guajira. Km 5,
vía a Maicao, Riohacha, Colombia
${ }^{2}$ Facultad de Ingeniería, Universidad del Zulia. Avenida 16 (Guajira) con Avenida Cecilio Acosta, Núcleo
Técnico, Maracaibo, Venezuela
*Autor para correspondencia: jpemon@uniguajira.edu.co

(Recibido mayo 2015; aceptado noviembre 2015)

Palabras clave: trazador, tiempo de retención hidráulico, flujo disperso, mezcla completa, zona muerta

\section{RESUMEN}

La hidráulica, junto con los procesos bioquímicos tiene una importancia esencial en la eficiencia de los sistemas de tratamiento biológico, tales como los reactores anaerobios de flujo ascendente (RAFA). En esta investigación se evaluó el desempeño hidráulico en un RAFA de 518 L utilizando un modelo de dispersión axial. El reactor fue inoculado con $105 \mathrm{~L}$ de lodo $(20 \% \mathrm{v} / \mathrm{v})$ y se alimentó con agua residual municipal del colector $\mathrm{C}$ de la ciudad de Maracaibo, Venezuela. Los ensayos fueron realizados al agregar una solución de $\mathrm{LiCl}$ de forma puntual en el efluente. $\mathrm{El} \mathrm{Li}^{+}$fue cuantificado como trazador. Las muestras fueron recolectadas en el efluente y en un punto intermedio del reactor a tiempos de retención hidráulica teóricos $\left(\mathrm{t}_{\mathrm{o}}\right)$ de $10,8,5,4$ y $3 \mathrm{~h}$ con tres entradas alineadas en el fondo del reactor y a $\mathrm{t}_{\mathrm{o}}$ de $5 \mathrm{~h}$ incrementando las entradas de forma impar hasta llegar a 15. El número de dispersión obtenido en la zona de lecho y manto de lodo (punto intermedio) fue mayor que en el efluente, obteniendo valores de $1.1842<$ $\mathrm{d}<1.7550$ y $0.2911<\mathrm{d}<0.7285$ respectivamente. El modelo de dispersión axial con inyección y recolección en flujo (IRF) e inyección en flujo y recolección en el fluido residente (IFRFR), presentó buenos ajustes a los datos experimentales de concentración del trazador. El reactor se comportó como mezcla completa en el lecho y manto de lodo y flujo disperso en el sedimentador.

Key words: tracer, hydraulic retention time, dispersed flow, complete mix, dead zone

\begin{abstract}
Hydraulics along with biogeochemical processes have a remarkable value in the efficiency of wastewater treatment systems as upflow anaerobic sludge reactors (UASB). In this study the hydraulic performance was evaluated on a UASB of $518 \mathrm{~L}$ volume capacity using an axial dispersion model. The reactor was inoculated with $105 \mathrm{~L}$ of sludge $(20 \% \mathrm{v} / \mathrm{v})$ and was fed with a local municipal wastewater flowing at the collector $\mathrm{C}$ of the city Maracaibo, Venezuela. Assays were carried out using a solution with $\mathrm{LiCl}\left(\mathrm{Li}^{+}\right.$measured as tracer) incorporated at the influent as instantaneous injection. Samples were collected at the effluent and at a midpoint of the reactor. Different theoretical hydraulic retention times $\left(\mathrm{t}_{\mathrm{o}}\right)$
\end{abstract}


were used such as 10, 8, 5, 4 and $3 \mathrm{~h}$ with three inputs aligned in the reactor bottom and $\mathrm{t}_{\mathrm{o}}$ $5 \mathrm{~h}$ increasing the inputs in odd numbers up to 15 . The number of dispersion obtained at the bed sludge blanket (midpoint) was higher than values obtained at the effluent varying from $1.1842<\mathrm{d}<1.7550$ and $0.2911<\mathrm{d}<0.7285$ respectively. The axial dispersion model with injection and detection in flux (IDF), and injection in flux and detection in resident fluid (IFDRF) showed a good fit from the experimental data of tracer concentration. Two defined areas were found at the reactor, one at the bed and sludge blanket behaving like complete stirred mixing tank and other at the settling basin as dispersed flow tank.

\section{INTRODUCCIÓN}

El reactor anaerobio de flujo ascendente (RAFA) es un reactor multifase compuesto por líquido-lodogas, donde sus interacciones determinan su comportamiento hidráulico. En este sistema los microorganismos actúan degradando los compuestos orgánicos presentes, para lo cual es necesario que ocurra un mezclado entre el substrato y los microorganismos. El diseño de un RAFA, al igual que los demás sistemas de tratamiento de aguas residuales, se ha centrado fundamentalmente en el proceso bioquímico. Se ha dejado en segundo plano los aspectos hidrodinámicos como las características de flujo, régimen de mezcla, tiempos de residencia, geometría del reactor, entre otros, lo que presenta una limitante en el diseño (Pérez y Torres 2008).

Ha sido tradicional el uso de los modelos unidimensionales para interpretar la distribución de los tiempos de residencia y los patrones de flujo en sistemas hidráulicos normalmente desde dos condiciones extremas: flujo pistón y mezcla completa, asignando un número de dispersión $\mathrm{d}<0.01$ para flujo pistón e infinito para mezcla completa (Levenspiel 1999, de Carvalvo 2006).

La evaluación hidráulica del RAFA data de los años 70, en Inglaterra. Desde entonces se estableció la dinámica del flujo considerando tres zonas: i) lecho en el cual existe una buena mezcla, espacios muertos, cortocircuito (desde el afluente hacia el manto), ii) manto como una región completamente mezclada (MC) y iii) sedimentador con presencia de flujo laminar descrito como flujo pistón (FP) (Heertjes y Van der Meer 1978, Bolle et al. 1986). Luego se supuso que no existe zona muerta en el lecho de lodo debido a que la generación de biogás hace que las zonas estancadas se mezclen (Van Der Meer y Heertjes 1983).

En el cuadro I se presenta un resumen de los resultados de los diferentes estudios hidráulicos realizado en RAFA.

Como se puede notar, hasta 1997 el RAFA fue dividido en tres zonas, año en el cual se inició la división en dos zonas: i) el lecho y manto de lodo considerado como flujo mezclado no ideal (por tener presencia de zonas muertas) y ii) el sedimentador descrito como flujo pistón con alto grado de dispersión. Además, existe un flujo advectivo directo del afluente al efluente (Wu y Hickey 1997). En la mayoría de los casos, el lecho y el manto han sido descritos como regiones mezcladas y el sedimentador como flujo pistón. Es importante aclarar que no existen rangos unificados del número de dispersión (d) para interpretar el tipo de flujo y, además, se han usado varios métodos para estimar dicha dispersión. El intercambio de elementos del fluido entre la zona activa e inactivas se conduce muy lentamente, de tal modo que los elementos del fluido que fueron atrapados llegan a tener largos tiempos de residencia (Giácoman et al. 2003).

En el lecho de lodo se puede presentar una zona de mezcla, pero también puede existir espacio muerto, flujo advectivo y flujo reverso (Heertjes y Van der Meer 1978), lo que resulta en una menor producción de biogás que origina una insuficiencia en el mezclado (Singh et al. 2006). La relación de flujo pistón sobre flujo mezclado $\left(\mathrm{I}_{\mathrm{P}} / \mathrm{I}_{\mathrm{M}}\right)$, es considerada como el parámetro de dinámica de fluidos que indica el nivel de flujo pistón en el reactor (Ren et al. 2008).

En esta investigación se evaluó el comportamiento hidráulico, flujo pistón, zonas muertas y mezcladas de un reactor anaerobio de flujo ascendente por medio de ensayos con trazador.

\section{MATERIALES Y MÉTODOS}

Unidad experimental, arranque y operación del RAFA. La investigación se llevó a cabo en un RAFA de 518 L construido en plástico transparente de $8 \mathrm{~mm}$. El reactor se alimentó con agua residual municipal del colector $\mathrm{C}$ de la ciudad de Maracaibo, Venezuela. Se inocularon $105 \mathrm{~L}$ de lodo $(20 \% \mathrm{v} / \mathrm{v})$ : $62 \mathrm{~L}$ provenientes de una cervecería local y $43 \mathrm{~L}$ de una laguna facultativa. La operación del reactor se llevó a cabo a diferentes tiempos de retención teóricos 
CUADRO I. PRINCIPALES RESULTADOS ENCONTRADOS EN LOS DIFERENTES MODELOS HIDRÁULICOS DE UN REACTOR ANAEROBIO DE FLUJO ASCENDENTE (RAFA)

\begin{tabular}{|c|c|c|c|c|}
\hline Fuente & No. de zonas & No. de dispersión, & Tipo de flujo & Escala \\
\hline $\begin{array}{l}\text { Heertjes y Van der Meer 1978, } \\
\text { Van der Meer y Heertjes 1983, }\end{array}$ & 3 & NR & $\begin{array}{l}\mathrm{LML}=\mathrm{MC} \\
\text { Sedimentador }=\mathrm{FP}\end{array}$ & Laboratorio, piloto y real \\
\hline Bolle et al. 1986 & 3 & NR & $\begin{array}{l}\mathrm{LML}=\mathrm{MC} \\
\text { Sedimentador }=\mathrm{FP}\end{array}$ & Piloto \\
\hline Wu y Hickey 1997 & 2 & 0.660 & $\begin{array}{l}\mathrm{LML}=\mathrm{MC} \\
\text { Sedimentador }=\mathrm{FP}\end{array}$ & Laboratorio \\
\hline Narnoli y Mehrotra 1997 & 3 & NR & & Laboratorio \\
\hline Singhal et al. 1998 & 2 & $\begin{array}{l}\operatorname{LML}(0.217<<10.0) \\
\mathrm{E}(0.263<<2.0)\end{array}$ & $\begin{array}{l}\mathrm{LM}=\mathrm{MC} \\
\text { Sedimentador }=\mathrm{FP}\end{array}$ & Laboratorio \\
\hline Lara et al. 2000 & NR & 0.289 & FP con alta dispersión & Real \\
\hline Avella 2001 & 2 & L y $M=N R$ y $E(0.38<<0.71)$ & $\begin{array}{l}\mathrm{LML}=\text { Mezclado } \\
\text { Sedimentador }=\text { FP }\end{array}$ & Real \\
\hline Zeng et al. 2005 & 2 & $\begin{array}{l}\operatorname{LML}(1.667<1.851) \\
\operatorname{E~}(0.123<\mathrm{d}<0.191)\end{array}$ & $\begin{array}{l}\mathrm{LML}=\text { Mezclado } \\
\text { Sedimentador }=\text { FP }\end{array}$ & Laboratorio \\
\hline Lou et al. 2006 & 2 & $\begin{array}{l}\operatorname{LML}(0.130<\mathrm{d}<0.227) \\
\mathrm{E}(0.120<\mathrm{d}<0.208)\end{array}$ & Pistón & Laboratorio \\
\hline Arroyave et al. 2005 & NR & 0.245 & Mezcla completa & Real \\
\hline de Carvalho et al. 2008 & NR & 0.112 y 0.121 & & Piloto \\
\hline
\end{tabular}

$\mathrm{NR}=$ No reporta, $\mathrm{LML}=$ lecho y manto de lodo, $\mathrm{E}=$ efluente, $\mathrm{MC}=$ mezcla completa

$\left(\mathrm{t}_{\mathrm{o}}\right): 10,8,5,4$ y $3 \mathrm{~h}$. Inicialmente el reactor operó con tres entradas alineadas y distribuidas en el fondo durante 334 días continuos y luego se modificó el sistema de distribución del flujo operando de 5 a 15 entradas por 113 días (Pérez y Aldana 2013; Fig. 1).

Ensayos hidráulicos. Para evaluar el comportamiento del flujo se realizaron ensayos hidráulicos utilizando una solución de $\mathrm{LiCl}$ y se midió la concentración de $\mathrm{Li}^{+}$como trazador por ser un elemento de poca interacción con el lodo (Lara et al. 2000, Avella 2001). Se agregaron $250 \mathrm{~mL}$ de una solución patrón de $5000 \mathrm{mgLi}^{+} / \mathrm{L}\left(\mathrm{C}_{0}=2.5 \mathrm{mg} / \mathrm{L}\right)$ de forma instantánea e inmediatamente se inició la recolección de las muestras en el efluente (inyección y recolección en flujo-IRF) y final del lecho y manto de lodo (inyección en el fluido y recolección en el fluido residente-IFRFR). La recolección se hizo por un periodo mínimo de 4 veces el $t_{0}$. Las mediciones de Li se realizaron en un espectrofotómetro de absorción atómica (marca Perkin Elmer, modelo 3110) de acuerdo con lo establecido en el estándar de métodos (APHA et al. 2005).

Cuando se evalúa hidráulicamente un sistema es necesario conocer la dispersión del flujo, razón por la cual en esta investigación se hizo una comparación del número de dispersión (d), calculado con los modelos populares y los obtenidos como parámetro de ajuste en un modelo propuesto. Para el primer caso se utilizaron las ecuaciones tradicionales de pequeña dispersión (PD), gran dispersión para recipiente abierto (GDra), gran dispersión para

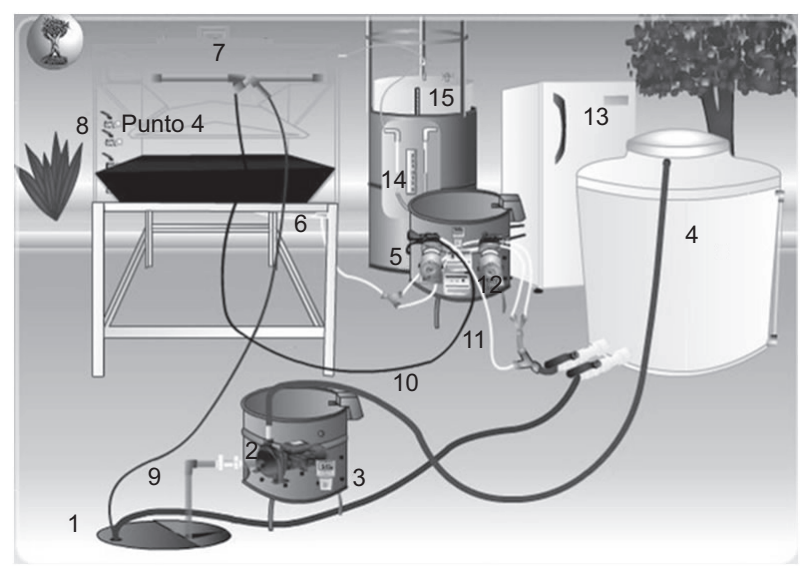

Fig. 1. Diagrama general del experimento. 1. Captación, 2. Bomba periférica, 3. Programador de horario, 4. Tanque de almacenamiento, 5. Bomba de alimentación, 6. Afluente, 7. Reactor, 8. Puntos de muestreo, 9. Efluente, 10. Muestra del efluente, 11. Bomba para muestreo, 13. Nevera para preservación, 14. Manómetro, 15. Medidor de biogás. 
recipiente cerrado (GDrc) conforme las ecuaciones 1-3, respectivamente (Levenspiel 1999), modelo de tanque en serie (TS) (Elgeti 1996) y pequeña dispersión para recipiente cerrado-abierto (PDrca) (Kim y Kim 1983), con las ecuaciones 4-5, respectivamente. Cabe resaltar que la ecuación 3 es la más utilizada en las mayoría de las investigaciones en RAFA(Morgan et al. 1997, Lara et al. 2000, Avella 2001, Arroyave et al. 2005) y en lagunas de estabilización (Bracho 2003, Aldana 2004).

Además de las ecuaciones anteriores, Pérez et al. (2015) propusieron un modelo que determina la dispersión, tiempo de retención hidráulico y velocidad ascensional del fluido. El modelo fue desarrollado utilizando ensayos hidráulicos con trazador inyectado en el afluente y recolectado en dos puntos del reactor i) en el efluente que corresponde a inyección y recolección en flujo (IRF) y ii) en un punto intermedio del reactor (punto 4 de la Fig. 1), que significa inyección en flujo y recolección en el fluido residente (IFRFR), tal como se muestra en las ecuaciones 6-7, respectivamente. Estas dos soluciones coincidieron con las citadas desde 1978 sin presentar su desarrollo (Kreft y Zuber 1978). Los parámetros hidráulicos obtenidos con estas ecuaciones se logran del ajuste de los datos experimentales al modelo, lo que indica que no es adimensional, como el caso de los modelos tradicionales (Pérez 2010).

Para los modelos populares, el coeficiente de dispersión fue obtenido con la ecuación 8 (Aldana 2004, Lou et al. 2006). La varianza se determinó de acuerdo con la ecuación 9 y para calcular el tiempo de retención hidráulico experimental ( $\overline{\mathrm{t}})$ se usó la ecuación 10 (Levenspiel 1999).

$$
\begin{aligned}
& \sigma_{\theta}^{2}=\frac{\sigma^{2}}{\overline{\mathrm{t}}^{2}}=2 \mathrm{~d} \\
& \sigma_{\theta}^{2}=\frac{\sigma^{2}}{\overline{\mathrm{t}}^{2}}=2 \mathrm{~d}+8 \mathrm{~d}^{2} \\
& \sigma_{\theta}^{2}=\frac{\sigma^{2}}{\overline{\mathrm{t}}^{2}}=2 \mathrm{~d}-2 \mathrm{~d}^{2}\left[1-\mathrm{e}^{-\left(\frac{1}{\mathrm{~d}}\right)}\right] \\
& \mathrm{d}=\frac{1}{2(\mathrm{~N}-1)} \\
& \sigma_{\theta}^{2}=2 \mathrm{~d}+3 \mathrm{~d}^{2}
\end{aligned}
$$$$
C(x, t)=\frac{M}{V} \frac{1}{\sqrt{4 \pi \frac{D}{v x}\left(\frac{t}{t}\right)^{3}}} e\left[-\frac{\left(1-\frac{t}{t}\right)^{2}}{4 \frac{D}{v x}\left(\frac{t}{t}\right)}\right]
$$

$$
\begin{aligned}
& C(x, t)=\frac{M}{V}\left(\frac{2}{\sqrt{4 \pi D t}} e^{-\frac{(x-v t)^{2}}{4 D t}}-\frac{v}{2 D} e^{\frac{v x}{D}} \operatorname{erfc}\left(\frac{v t+x}{\sqrt{4 D t}}\right)\right) \\
& D=d v x \\
& \sigma^{2}=\frac{\sum t^{2} \Delta t}{\sum C \Delta t}-\bar{t}_{t}^{2} \\
& \bar{t}_{t}=\frac{\sum \mathrm{tC} \Delta t}{\sum C \Delta t}
\end{aligned}
$$

Donde: $\sigma_{\theta}^{2}=$ varianza adimensional de la curva de distribución del trazador, $\mathrm{d}=$ número de dispersión (adimensional), $\mathrm{D}=$ coeficiente de dispersión axial $\left(\mathrm{m}^{2} / \mathrm{min}\right), \overline{\mathrm{t}}=$ tiempo de retención hidráulico $(\mathrm{min})$, $\Delta \mathrm{t}=$ intervalo de tiempo entre toma de muestra ( $\mathrm{min}$ ), $\mathrm{t}$ = tiempo transcurrido desde la inyección del trazador hasta la toma de la muestra ( $\mathrm{min}), \mathrm{x}=$ altura entre el punto de inyección y recolección del trazador $(\mathrm{m}), \mathrm{C}=$ concentración del trazador $(\mathrm{mg} / \mathrm{L}), \mathrm{M}=$ masa del trazador inyectada $(\mathrm{mg}), \mathrm{V}=$ volumen del reactor $(\mathrm{L}), \mathrm{v}=$ velocidad ascensional del fluido $(\mathrm{m} /$ $\min ), \overline{\mathrm{t}}_{\mathrm{t}}=$ tiempo de retención hidráulico calculado con la ecuación tradicional (min).

Para analizar el patrón hidráulico del RAFA se usaron las ecuaciones 11-14. La eficiencia hidráulica, la fracción del volumen pistón, la fracción de volumen muerto (Bolle et al. 1986, Morgan et al. 1997, Martin 2000, Ren et al. 2008) y la fracción de volumen mezclado (Martin 2000, Ren et al. 2008) están contempladas en dichas ecuaciones.

$$
\begin{aligned}
& \beta=\frac{\mathrm{t}}{\mathrm{t}_{\mathrm{o}}} \\
& \mathrm{I}_{\mathrm{p}}=\frac{\mathrm{V}_{\mathrm{p}}}{\mathrm{V}}=\frac{\theta_{\text {max }}}{\bar{\theta}} \\
& \mathrm{I}_{\mathrm{m}}=\frac{\mathrm{V}_{\mathrm{m}}}{\mathrm{V}}=1-\beta \\
& \mathrm{I}_{\mathrm{M}}=\frac{\mathrm{V}_{\mathrm{M}}}{\mathrm{V}}=1-\frac{\mathrm{V}_{\mathrm{p}}}{\mathrm{V}}-\frac{\mathrm{V}_{\mathrm{m}}}{\mathrm{V}}
\end{aligned}
$$

Donde:

$\beta=$ eficiencia hidráulica (adimensional), $\mathrm{t}_{\mathrm{o}}=$ tiempo de retención hidráulico teórico $(\mathrm{h})$, $\mathrm{V}_{\mathrm{p}}=$ volumen de flujo pistón $(\mathrm{L}), \mathrm{V}_{\mathrm{m}}=$ volumen 
muerto $(\mathrm{L}), \mathrm{I}_{\mathrm{p}}=$ fracción de volumen pistón, $\mathrm{I}_{\mathrm{m}}=$ fracción de volumen muerto, $\mathrm{I}_{\mathrm{M}}=$ fracción de volumen mezclado (adimensional), $\theta_{\max }=$ tiempo adimensional para la máxima concentración, $\bar{\theta}=$ tiempo de retención hidráulico adimensional, $\mathrm{V}=$ volumen del reactor $(\mathrm{L}), \overline{\mathrm{t}}=$ tiempo de retención hidráulico $(\mathrm{min})$.

\section{RESULTADOS Y DISCUSIÓN}

El reactor fue inoculado y se dejó con carga (lote) durante 7 días, tiempo en que se logró una eficiencia de remoción del $70 \%$ en términos de la DQO total $\left(\mathrm{DQO}_{\mathrm{T}}\right)$. Al octavo día se inició la operación a flujo continuo. Las eficiencias de remoción obtenidas en cuanto a $\mathrm{DQO}_{\mathrm{T}}$ fueron de $33.12 \%$ y de DQO soluble (DQOs) de $24.95 \%$, lo que hizo más evidente la necesidad de una mejor distribución del afluente aumentando el número de entradas de 3 a 15. Durante la operación, la eficiencia de remoción (DQO) permaneció cerca al $30 \%$ cuando el reactor funcionó con tres entradas y se incrementó con el número de entradas hasta un máximo de $62.49 \%$ y $62.22 \%$ para la $\mathrm{DQO}_{\mathrm{T}}$ y $\mathrm{DQO}_{\mathrm{S}}$, respectivamente.
La temperatura promedio del afluente fue de 33.93 $\pm 1.39{ }^{\circ} \mathrm{C}$ y del efluente $31.62 \pm 1.83^{\circ} \mathrm{C}$. El pH del afluente $(7.19 \pm 0.19$ y $7.26 \pm 0.18)$ fue ligeramente superior al efluente $(6.92 \pm 0.13$ y $6.90 \pm 0.18)$, en ambos casos permaneció cerca del rango de la neutralidad, el cual es recomendado para un buen desarrollo de la digestión anaerobia (Aiyuk et al. 2006, Álvarez et al. 2006, Gallegos et al. 2010). Lo anterior indica que la alcalinidad del sistema (236 \pm $33.64 \mathrm{mgCaCO}_{3} / \mathrm{L}$ ) fue suficiente para mantener la estabilidad en el $\mathrm{pH}$. La producción de biogás varió de 0.165 a $0.456 \mathrm{~m}^{3}$ biogás/ $\mathrm{kgDQO}$, con un promedio de $0.280 \mathrm{~m}^{3}$ biogás $/ \mathrm{kgDQO}$, resultado similar a los obtenidos a escala real (Peña et al. 2006) de 0.341 a $0.434 \mathrm{~m}^{3}$ biogás $/ \mathrm{kgDQO}$ removida. El porcentaje de metano varió entre $49.4 \%$ y $88.92 \%$ con un promedio de $72.32 \%$, estos resultados son similares a los encontrados a escala de laboratorio (Bermúdez et al. 2003).

Como se puede observar en el cuadro II, el número de dispersión obtenido por los métodos tradicionales difiere del obtenido para las dos condiciones del modelo propuesto (IRF e IFRFR). Esta contradicción se debe a que las ecuaciones tradicionales no

CUADRO II. NÚMEROS DE DISPERSIÓN POR MÉTODOS TRADICIONALES Y MODELO MATEMÁTICO

\begin{tabular}{|c|c|c|c|c|c|c|c|c|c|c|c|c|}
\hline \multirow{2}{*}{$\begin{array}{l}\mathrm{t}_{\mathrm{o}} \\
\text { (h) }\end{array}$} & \multirow{2}{*}{$\begin{array}{c}\text { No. } \\
\text { entradas }\end{array}$} & \multicolumn{5}{|c|}{ Número de dispersión (d) por métodos tradicionales } & \multicolumn{2}{|c|}{ Modelo de dispersión } & \multicolumn{4}{|c|}{ Ajuste modelo de dispersión } \\
\hline & & $\mathrm{PD}$ & PDrca & GDrc & GDra & TS & $\mathrm{d}$ & $\mathrm{D}\left(\mathrm{m}^{2} / \mathrm{h}\right)$ & $\mathrm{R}^{2}$ & $\mathrm{R}_{\mathrm{a}}^{2}$ & SSE & RMSE \\
\hline \multicolumn{13}{|c|}{ Efluente MF en operación con 3 entradas (IRF) } \\
\hline 10 & 3 & 0.2550 & 0.1969 & 0.4053 & 0.1567 & 0.5204 & 0.2911 & $8.662 \mathrm{E}-03$ & 0.9995 & 0.9995 & 0.0335 & 0.0198 \\
\hline 8 & 3 & 0.2946 & 0.2212 & 0.5417 & 0.1738 & 0.7171 & 0.3828 & & 0.9990 & 0.9990 & 645 & 0.0279 \\
\hline 5 & 3 & 0.1885 & 0.1533 & 0.2498 & 0.1255 & 0.3026 & 0.4209 & -02 & 0.9835 & 0.9833 & 1.2090 & 0.1229 \\
\hline $5^{*}$ & 3 & 0.2711 & 0.2069 & & & 0.5 & 0.7 & & & 0.9906 & & \\
\hline 4 & 3 & 0.2085 & & & & & 0.5 & & & 0.9909 & & 279 \\
\hline $3 *$ & 3 & 0.2117 & 0.1689 & 0.2968 & 0.1368 & 0.3673 & 0.3544 & 4.951E-02 & 0.9908 & 0.9907 & 1.1104 & 0.1205 \\
\hline \multicolumn{13}{|c|}{ Zona del lecho y manto de lodo MF en operación con 3 entradas (IFRFR) } \\
\hline 8 & 3 & 0.5373 & 0.3517 & 5.0000 & 0.2622 & 5.0000 & 1.3736 & 4.177E-02 & 0.9985 & & & 0.0668 \\
\hline 5 & 3 & 0.1067 & & & & & & & & & & \\
\hline $5 *$ & 3 & 0.1360 & 0.3065 & 2.9126 & 0.2320 & 1.1176 & 1.184 & E-01 & 0.9899 & 0.9897 & 1.7145 & 0.1962 \\
\hline 4 & 3 & 0.3562 & 0.2570 & 0.8960 & 0.1985 & 1.2379 & 1.3879 & E-01 & 0.9989 & 0.9989 & 0.4061 & 0.0797 \\
\hline 3 & 3 & 0.3983 & 0.2804 & 1.3806 & 0.2144 & 1.9586 & 1.7550 & 2.5981 & 0.9793 & 0.9790 & 1.7701 & 0.2459 \\
\hline \multicolumn{13}{|c|}{ Efluente MF con modificaciones (IRF) } \\
\hline 5 & 5 & 0.3424 & 0.2492 & 0.7935 & 0.1932 & 1.08641 & 0.3369 & $1.952 \mathrm{E}-02$ & 0.9936 & 0.9936 & 0.4338 & 0.0741 \\
\hline 5 & 7 & 0.3536 & 0.2556 & 0.8755 & 0.1975 & 1.2076 & 0.4429 & $2.537 \mathrm{E}-02$ & 0.9957 & 0.9957 & 0.2634 & 0.0570 \\
\hline 5 & 9 & 0.3745 & 0.2673 & 1.0675 & 0.2055 & 1.4924 & 0.4211 & $2.598 \mathrm{E}-02$ & 0.9902 & 0.9901 & 0.6521 & 0.0903 \\
\hline 5 & 11 & 0.3444 & 0.2504 & 0.8070 & 0.1939 & 1.1066 & 0.4431 & $2.995 \mathrm{E}-02$ & 0.9958 & 0.9957 & 0.2826 & 0.0598 \\
\hline 5 & 13 & 0.3949 & 0.2785 & 1.3265 & 0.2131 & 1.8780 & 0.7285 & 4.049E-02 & 0.9865 & 0.9863 & 1.2800 & 0.1265 \\
\hline 5 & 15 & 0.3896 & 0.2756 & 1.2500 & 0.2112 & 1.7639 & 0.6680 & $4.599 \mathrm{E}-02$ & 0.9866 & 0.9862 & 1.0740 & 0.1166 \\
\hline
\end{tabular}

${ }^{*}$ = ensayo réplica, $\mathrm{t}_{\mathrm{o}}=$ tiempo de retención hidráulico teórico, $\mathrm{PD}=$ pequeña dispersión, $\mathrm{PDrca}=$ pequeña dispersión para recipiente cerrado, GDrc = gran dispersión para recipiente cerrado, GDra = gran dispersión par recipiente abierto, TS $=$ tanque en serie, $\mathrm{d}=$ número de dispersión, $\mathrm{R}^{2}=$ coeficiente de determinación, $\mathrm{R}_{\mathrm{a}}^{2}=$ coeficiente de determinación ajustado, $\mathrm{SSE}=$ sumatoria de los errores al cuadrado, $\mathrm{RMSE}=$ raíz del error cuadrado medio, $\mathrm{IRF}=$ inyección y recolección en flujo, IFRFR = inyección en flujo y recolección en el fluido residente 
consideran las condiciones reales de aplicación del trazador y las condiciones de frontera. Una gran contradicción se observó en los resultados de un estudio realizado a escala piloto por de Carvalho (2008), cuando se usaron las ecuaciones propuestas por Levesnpiel en 1999 y se obtuvieron valores mayores para el modelo de gran dispersión y recipiente abierto (GDra) comparados con el de pequeña dispersión (PD). También se encontraron valores de dispersión diferentes con los mismos datos experimentales (reactor escala de laboratorio), los cuales fueron menores cuando se usó la ecuación de GDra (Lou et al. 2006) y mayores al definir condiciones de borde adecuadas (Zeng et al. 2005), del mismo modo que se observó en un estudio a escala real (Avella 2001, Peña et al. 2006). Lo anterior permite afirmar que se pueden obtener resultados contradictorios si no se consideran las condiciones de borde reales. Es importante aclarar que todas las ecuaciones tradicionales determinan el número de dispersión o el coeficiente de dispersión a través de la varianza, lo que no es recomendado estadísticamente cuando se tienen curvas de distribución del trazador asimétricas a la derecha (Devore 2001). Una verificación de las bondades de ajuste entre los métodos tradicionales y los modelos IRF e IFRFR fue realizada previamente (Pérez 2010). Posteriormente se verificó en un reactor anaerobio con doble cámara confirmando las bondades de dichos modelos (Rincón et al. 2011).

Cuando el reactor funcionó con tres entradas alineadas en el fondo, el número de dispersión obtenido en la zona de lecho y manto de lodo (LML) fue mayor que en el efluente, registrando valores de $1.1842<$ $\mathrm{d}<1.7550$ y $0.2911<\mathrm{d}<0.7036$, respectivamente. Valores similares fueron registrados cuando se realizaron las modificaciones en el número de entradas, con número de dispersión $0.3369<\mathrm{d}<0.7285$. El mayor valor en la zona del lecho y manto de lodo se debió a una mejor distribución del afluente que generó un incremento en la tasa de flujo de biogás produciendo una mayor turbulencia, tal como se reportó en un lecho fluidizado (Kim y Kim 1983), en un RAFA a escala real (Peña et al. 2006) y a escala de laboratorio (Morgan et al. 1997). Por lo anterior, se puede concluir que en la zona del lecho y manto de lodo el reactor describió un comportamiento de mezcla completa y a nivel general registró flujo disperso.

En la figura 2 se muestran seis ajustes del modelo propuesto a los datos experimentales de concentración del trazador para $\mathrm{t}_{\mathrm{o}}$ de 8 y $5 \mathrm{~h}$, en ningún caso se observó presencia de flujo advectivo. La figura 2a y $\mathbf{2 b}$ corresponden a muestras tomadas en el efluente cuando el reactor funcionó con tres entradas en el sistema de distribución del flujo. La figura 2 c y 2 d corresponden a las mismas condiciones pero las muestras fueron recolectadas en el punto intermedio, el cual representa el final del lecho y manto de lodo. Las figuras $2 \mathrm{e}$ y $\mathbf{2 f}$ corresponden a $\mathrm{t}_{\mathrm{o}}$ de $5 \mathrm{~h}$, tomadas en el efluente operando con cinco y 11 entradas, respectivamente.

Se encontró una correlación lineal positiva entre el coeficiente de dispersión (D) y la velocidad de ascenso tanto en el punto intermedio del reactor (final del manto de lodo) como en el efluente (a nivel general del reactor) con $\mathrm{R}=0.9754\left(\mathrm{R}^{2}=0.9514\right)$ y 0.9688 $\left(\mathrm{R}^{2}=0.9387\right)$, respectivamente (Fig. 3a). El valor de $\mathrm{D}$ también presentó una correlación lineal positiva $\left(\mathrm{R}=0.9670, \mathrm{R}^{2}=0.9352\right)$ con el número de entradas como se observa en la figura $\mathbf{3 b}$, lo que se debió a una mejor distribución del efluente, aumentando la eficiencia de degradación y la producción de biogás. Así, se demostró que una mejor distribución del afluente es esencial para obtener mayores eficiencias en la remoción de materia orgánica.

Patrón hidráulico. En el cuadro III se presentan las condiciones de operación y los parámetros relacionados con el patrón de flujo. Como se puede observar, las eficiencias hidráulicas para la fase líquida fueron cercanas a la unidad en todos los casos. El alto porcentaje de trazador recuperado garantiza una confiabilidad en los resultados obtenidos a partir de los ensayos hidráulicos.

Cuando el reactor funcionó con tres entradas, la zona muerta varió de $0.049<\mathrm{I}_{\mathrm{m}}<0.285$ para el reactor en general y $0.505<\mathrm{I}_{\mathrm{m}}<0.693$ para el LML; estas zonas aumentaron con el incremento de la altura del lecho de lodo $\left(12.6 \mathrm{~cm}<\mathrm{H}_{\mathrm{L}}<20.1 \mathrm{~cm}\right)$. La velocidad teórica fue mayor que la velocidad calculada a partir del modelo propuesto como parámetro de ajuste debido a que la primera se determinó sin considerar el volumen ocupado por el lodo como se hace de manera tradicional al dividir el volumen del reactor entre el tiempo de retención. Si bien el lodo es una masa acuosa, parte de él ocupa un espacio dentro del reactor conocido como zona muerta biológica que explica el mayor valor de zonas estancadas en la parte inferior del reactor. La diferencia en las velocidades indica que el agua viajó más rápido en el LML que en el sedimentador. Es importante notar que existe una diferencia de densidades entre el lodo y el líquido, que genera cambios de comportamiento del fluido ascendente (Gimenez et al. 2002). Lo anterior confirma que no existe una velocidad uniforme debido a la presencia de flujo advectivo, de zonas muertas (o regiones estancadas) o de volumen de lodo. Las fluctuaciones de velocidad también pueden ser 

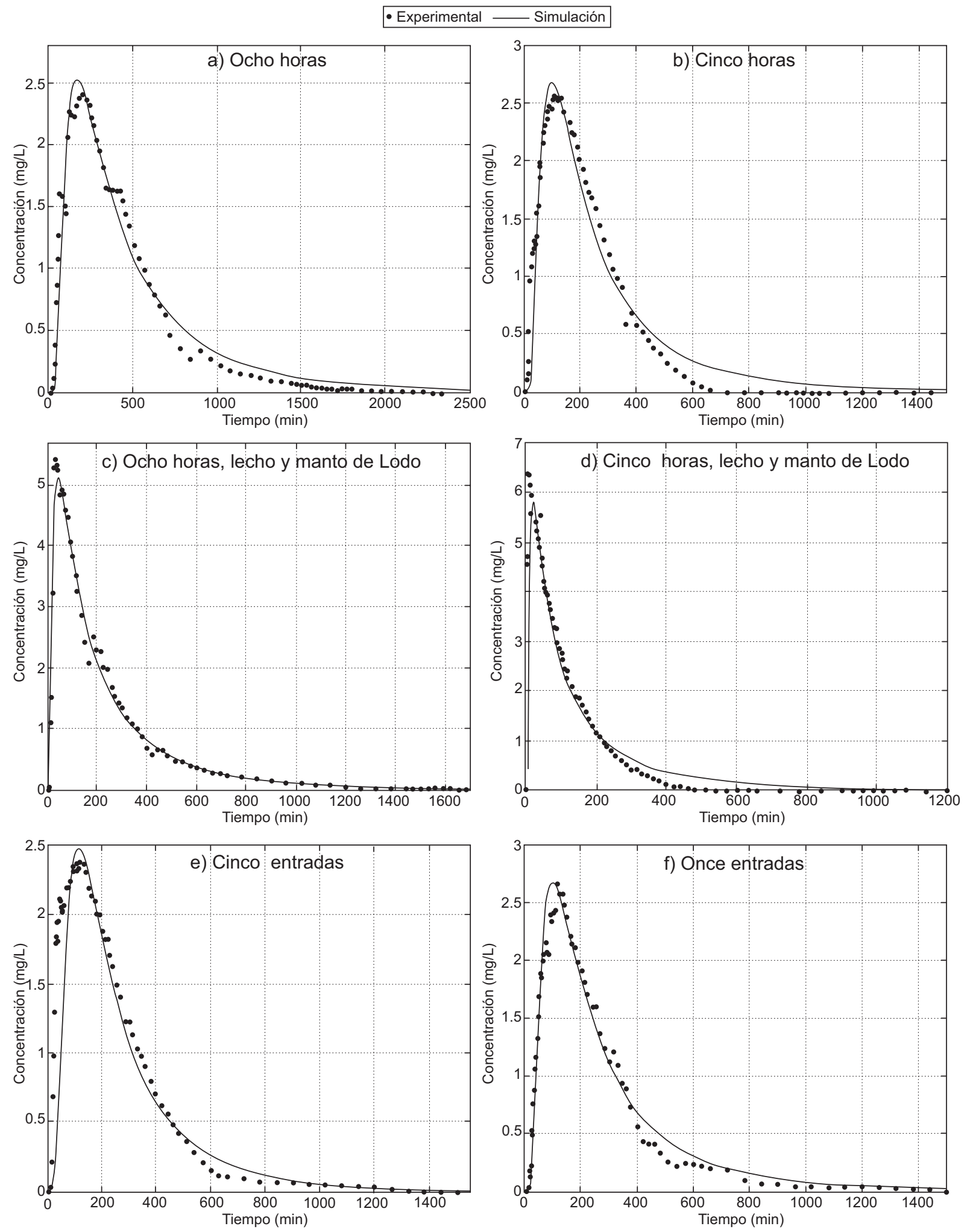

Fig. 2. Ajuste del modelo propuesto a los datos experimentales de concentración del trazador. a) a las 8 h, b) a las 5 h, c) $8 \mathrm{~h}$, lecho y manto de lodo, d) 5 h, lecho y manto de lodo, e) 5 entradas, f) 11 entradas 

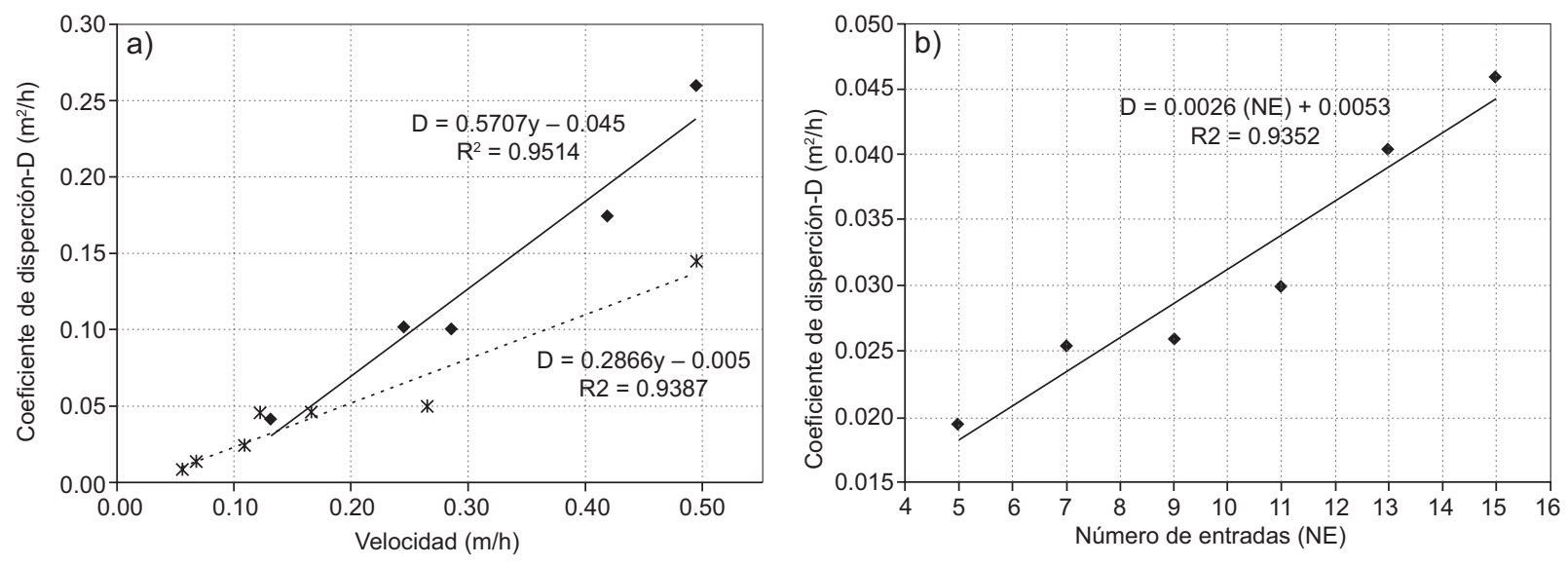

- Lecho y manto de lodo $\cdots \cdot$... Efluente

Fig. 3. Comportamiento del coeficiente de dispersión (D) con la velocidad y el número de entradas del sistema de distribución del flujo. a) D vs. velocidad, b) D vs. número de entradas

generadas por la turbulencia de difusión molecular en dirección al flujo. Estos fenómenos actúan ya sea por sí solos o en combinación y contribuyen con la dispersión axial haciendo que los patrones de mezclado se desvíen de las condiciones ideales (Iliuta et al. 1998). Es importante mencionar que al inicio de la operación el reactor tenía una relación de lodo granular/floculante de 60/40, la cual disminuyó sustancialmente a través

CUADRO III. CARACTERÍSTICAS HIDRÁULICAS DEL FLUJO EN EL REACTOR

\begin{tabular}{|c|c|c|c|c|c|c|c|c|c|c|c|c|}
\hline \multirow[t]{2}{*}{ Entradas } & \multirow{2}{*}{$\begin{array}{c}\mathrm{t}_{\mathrm{o}} \\
(\mathrm{min})\end{array}$} & \multirow{2}{*}{$\begin{array}{l}\mathrm{H}_{\mathrm{L}} \\
(\mathrm{m})\end{array}$} & \multirow{2}{*}{$\begin{array}{c}\mathrm{Q}_{\mathrm{g}} \\
(\mathrm{L} / \mathrm{h})\end{array}$} & \multirow{2}{*}{$\begin{array}{c}\mathrm{v}_{\mathrm{t}} \\
(\mathrm{m} / \mathrm{h})\end{array}$} & \multirow{2}{*}{$\begin{array}{c}\% \\
\mathrm{Li}^{+}\end{array}$} & \multicolumn{7}{|c|}{ Modelo de dispersión } \\
\hline & & & & & & $\overline{\mathrm{t}}(\mathrm{min})$ & $\mathrm{v}(\mathrm{m} / \mathrm{h})$ & $\beta=\mathrm{t}_{0} / \mathrm{t}$ & $\bar{\theta}=\bar{t}_{\mathrm{t}} / \overline{\mathrm{t}}$ & $\mathrm{I}_{\mathrm{p}}$ & $\mathrm{I}_{\mathrm{m}}$ & $\mathrm{I}_{\mathrm{M}}$ \\
\hline \multicolumn{13}{|c|}{ Efluente MF en operación con 3 entradas (IRF) } \\
\hline 3 & 624.0 & 12.6 & 1.22 & 0.0507 & 98.7 & 560.0 & 0.0565 & 0.897 & 0.988 & 0.551 & 0.103 & 0.347 \\
\hline 3 & 484.3 & 13.5 & 2.56 & 0.0652 & 101.7 & 460.8 & 0.0685 & 0.951 & 0.933 & 0.436 & 0.049 & 0.516 \\
\hline 3 & 307.8 & 13.8 & 3.16 & 0.1029 & 92.3 & 288.7 & 0.1097 & 0.938 & 0.712 & 0.479 & 0.062 & 0.459 \\
\hline 3 & 300.1 & 13.8 & 3.28 & 0.1057 & 93.3 & 260.0 & 0.1221 & 0.866 & 0.663 & 0.241 & 0.134 & 0.625 \\
\hline 3 & 243.3 & 13.9 & 3.82 & 0.1297 & 95.4 & 190.0 & 0.1661 & 0.781 & 0.677 & 0.456 & 0.219 & 0.325 \\
\hline 3 & 183.6 & 14.1 & 4.08 & 0.1715 & 91.9 & 120.2 & 0.2641 & 0.715 & 1.034 & 0.353 & 0.285 & 0.362 \\
\hline 3 & 185.1 & 20.1 & 3.68 & 0.3842 & 93.2 & 64.0 & 0.4959 & 0.775 & 0.904 & 0.589 & 0.225 & 0.186 \\
\hline \multicolumn{13}{|c|}{ Zona del lecho y manto de lodo MF en operación con 3 entradas (IFRFR) } \\
\hline 3 & 278.9 & 13.5 & 2.56 & 0.0646 & 98.9 & 129.7 & 0.1310 & 0.465 & 1.929 & 0.056 & 0.535 & 0.409 \\
\hline 3 & 176.5 & 13.8 & 3.16 & 0.1020 & 97.4 & 73.3 & 0.2456 & 0.415 & 1.427 & 0.004 & 0.585 & 0.411 \\
\hline 3 & 172.8 & 13.8 & 3.28 & 0.1042 & 81.8 & 59.1 & 0.2854 & 0.342 & 1.586 & 0.055 & 0.658 & 0.287 \\
\hline 3 & 140.1 & 13.9 & 3.82 & 0.1285 & 72.6 & 43.0 & 0.4182 & 0.307 & 1.453 & 0.015 & 0.693 & 0.293 \\
\hline \multicolumn{13}{|c|}{ Efluente MF con modificaciones (IRF) } \\
\hline 5 & 298.5 & 15.5 & 4.34 & 0.1045 & 100.7 & 280.0 & 0.1114 & 0.938 & 0.876 & 0.459 & 0.062 & 0.479 \\
\hline 7 & 298.1 & 16.1 & 4.36 & 0.1041 & 93.0 & 280.0 & 0.1108 & 0.939 & 0.884 & 0.303 & 0.061 & 0.636 \\
\hline 9 & 294.3 & 16.7 & 5.09 & 0.1054 & 91.1 & 259.9 & 0.1194 & 0.883 & 0.913 & 0.261 & 0.117 & 0.622 \\
\hline 11 & 299.7 & 17.1 & 5.89 & 0.1041 & 98.1 & 240.0 & 0.1300 & 0.801 & 1.104 & 0.347 & 0.199 & 0.453 \\
\hline 13 & 298.9 & 17.8 & 6.06 & 0.1050 & 96.8 & 295.3 & 0.1063 & 0.988 & 0.777 & 0.220 & 0.012 & 0.768 \\
\hline 15 & 302.3 & 18.9 & 6.12 & 0.1044 & 91.8 & 241.1 & 0.1309 & 0.798 & 0.890 & 0.334 & 0.202 & 0.463 \\
\hline 15 & 239.3 & 17.5 & 4.03 & 0.1310 & 89.3 & 152.9 & 0.2051 & 0.639 & 1.073 & 0.268 & 0.361 & 0.370 \\
\hline
\end{tabular}

$\mathrm{NA}=$ No aplica, $\mathrm{MF}=$ modelo físico, $\mathrm{H}_{\mathrm{L}}=$ altura del lecho de lodo, $\mathrm{Q}_{\mathrm{g}}=$ caudal de biogás, $\mathrm{v}_{\mathrm{t}}=$ velocidad ascensional del flujo teórica, $\% \mathrm{Li}^{+}=$trazador recuperado $(\%), \overline{\mathrm{t}}=$ Tiempo de retención hidráulico calculado con el modelo propuesto (ecuaciones 6 ó 7), $\mathrm{t}_{\mathrm{o}}=$ tiempo de retención hidráulico teórico, $\overline{\mathrm{t}}_{\mathrm{t}}=$ tiempo de retención hidráulico calculado con la ecuación tradicional (ecuación 10), $\mathrm{v}=$ velocidad calculada con el modelo propuesto, $\beta=$ eficiencia hidráulica, $\bar{\theta}=$ tiempo de retención hidráulico adimensional, Ip $=$ fracción de volumen pistón, $\mathrm{I}_{\mathrm{m}}=$ fracción de volumen muerto, $\mathrm{I}_{\mathrm{M}}=$ fracción de volumen mezclado (adimensional). IRF = inyección y recolección en flujo, IFRFR = inyección en flujo y recolección en el fluido residente 
del tiempo de operación. Al momento de hacer las modificaciones al sistema de distribución del flujo (transcurrido 11 meses) la relación fue de 10/90.

Luego de las modificaciones (5-15 entradas) se obtuvieron $0.012<\mathrm{I}_{\mathrm{m}}<0.202$, aumentando cuando el sistema operó con 15 entradas. Esta disminución del volumen muerto hizo que se incrementara el flujo mezclado $\left(0.463<\mathrm{I}_{\mathrm{M}}<0.636\right)$, lo anterior comprueba que el mezclado y la distribución del flujo es esencial en este proceso, tal como lo han afirmado otras investigaciones (Avella 2001, Morel et al. 2004). Los valores encontrados para $\mathrm{I}_{\mathrm{p}}, \mathrm{I}_{\mathrm{m}}, \mathrm{I}_{\mathrm{M}}$, son similares a los encontrados en un reactor a escala real (Avella 2001).

En un filtro anaerobio, Young y Young (1998) encontraron fracción de zona muerta $\left(\mathrm{I}_{\mathrm{m}}\right)$ que variaron de 0.5 a 0.75 . Grobicki y Stuckey (1992) consideraron como muy alto un valor $\mathrm{I}_{\mathrm{m}}$ de $0.22 \mathrm{en}$ un reactor anaerobio con deflectores. En un RAFA a escala de laboratorio Morgan et al. (1997) modificaron la altura del colector de biogás (operando con agua y lodo) de 15 a $9.5 \mathrm{~cm}$ medidos desde el fondo del reactor, encontrando que el valor de la zona muerta se redujo de $0.13<\mathrm{I}_{\mathrm{m}}<-0.52$. También agregaron un flujo de aire de $411 \mathrm{~mL} / \mathrm{min}$ (operando con agua, lodo y gas) y la zona muerta varió de $0.34<\mathrm{I}_{\mathrm{m}}<0.38 \mathrm{y}$ al incrementarse a $626 \mathrm{~mL} / \mathrm{min}$ permaneció de $0.43<\mathrm{I}_{\mathrm{m}}<0.34$. Los investigadores consideraron que este comportamiento se debió al mezclado originado por el aire. Morgan et al. (1997) no explicaron el valor negativo encontrado $(-0.52)$, lo que realmente significa ausencia de zonas muertas y por el contrario indica que existió retroflujo en el sistema (Aldana 2004).

Morgan et al. 1997, también realizaron ensayos en un RAFA a escala piloto ( $840 \mathrm{~L}$ ) con un flujo de aire de $15 \mathrm{~m} 3 / \mathrm{d}$ (equivalentes a una producción de $0.22 \mathrm{~m}^{3} \mathrm{CH}_{4} / \mathrm{kgDQOr}$ ) encontrando que el colector de biogás, ejerció una gran influencia en la fracción de volumen muerto, el cual fue de 0.28 sin colector y disminuyó a $0.20,0.016$ y -0.09 cuando el colector se ubicó a 3.17 m, $1.47 \mathrm{~m}$ y $0.82 \mathrm{~m}$ respectivamente.

Resultados similares fueron encontrados por Ren et al. (2008) en un RAFA de 7.5 L, donde obtuvieron $0.00<\mathrm{I}_{\mathrm{m}}<0.17,0.36<\mathrm{I}_{\mathrm{p}}<0.45$ y de $0.48<\mathrm{I}_{\mathrm{M}}<$ 0.62 . En un RAFA de $8.6 \mathrm{~L}$ se determinaron valores de $\mathrm{I}_{\mathrm{m}}$ de 0.12 y 0.29 para tiempos de retención de $3 \mathrm{y}$ $12 \mathrm{~h}$, respectivamente (Ji et al. 2014). Wu y Hickey (1997) reportan un predominio de flujo mezclado $\left(\mathrm{I}_{\mathrm{M}}\right.$ $=0.91)$ y bajos valores de flujo pistón y zona muerta $\left(\mathrm{I}_{\mathrm{p}}=0.02, \mathrm{I}_{\mathrm{m}}=0,07\right)$ en un RAFA de $3.1 \mathrm{~L}$. Heertjes y Van der Meer (1978) encontraron resultados parecidos se encontraron a escala real, $\mathrm{I}_{\mathrm{p}}=0.005, \mathrm{I}_{\mathrm{M}}=0.95 \mathrm{y}$ ausencia de zonas muertas.

\section{CONCLUSIONES}

El número de dispersión obtenido con los métodos tradicionales difiere del logrado con el modelo propuesto (IRF y IFRFR), el cual presentó un buen ajuste a los datos experimentales.

El número de dispersión obtenido en todos los modelos fue mayor a 0.10 , lo que indica que no ocurrió flujo pistón en el reactor y por el contrario, según el modelo propuesto, en la zona del lecho y manto de lodo ocurrió un flujo mezclado. A nivel general el rector registró flujo disperso.

El coeficiente de dispersión se incrementó de forma proporcional con el aumento de velocidad y el número de entradas del sistema de distribución del flujo, tanto en la zona del lecho y manto de lodo como en el efluente, lo que se debió a un mejor mezclado y con ello se logró un buen contacto entre microorganismo y sustrato.

La fracción de zonas muertas estimadas en la zona del lecho y manto de lodo fueron mayores a las estimadas en el efluente, debido a la zona muerta biológica que está presente en esta parte del reactor.

El reactor está compuesto por dos zonas, la zona 1 que representa el lecho y manto de lodo que describió un comportamiento de mezcla completa con presencia de zonas muertas y la zona 2 con el sedimentador que se comporta como flujo disperso.

\section{AGRADECIMIENTOS}

A la Universidad La Guajira, al Departamento de Ingeniería Sanitaria y Ambiental (DISA) por el apoyo financiero, al Centro de Investigaciones del Agua (CIA) de la Universidad del Zulia por su apoyo logístico.

\section{REFERENCIAS}

Aiyuk S., Forrez L., De Kempeneer L., Van Haandel A. y Verstraete W. (2006). Anaerobic and complementary treatment of domestic sewage in regions with hot climates-A review. Bio-Technol. 97, 2225-2241. DOI: 10.1016/j.biortech.2005.05.015.

Aldana G.J. (2004). Hydraulic behaviour and performance improvement of waste stabilization ponds using a computational fluid dynamic and physical model. Tesis de Doctorado. Universidad de Surrey, Inglaterra, 290 pp.

Álvarez J.A., Ruíz I., Gómez M., Presas J. y Soto M. (2006). Start-up alternatives and performance of an UASB pilot plant treating diluted municipal 
wastewater at low temperature. Bio-Technol. 97, 1640-1649. DOI: 10.1016/j.biortech.2005.07.033.

APHA, AWWA y WEF (2005). Standard Methods for the Examination of Water and Wastewater. 20a ed. American Public Health Association, American Water Works Association y Water Environment Federation. Washington, EUA, 1325pp

Arroyave D., González M. y Gallego D. (2005). Evaluación del comportamiento hidráulico de un reactor UASB utilizado para el tratamiento de aguas residuales. Memorias. VIII Congreso Peruano de ingeniería química. Lima, Perú. 24 al 27 de abril, pp. 45-55.

Avella G.P. (2001). Evaluación del comportamiento hidrodinámico de un reactor UASB y su influencia en la remoción de materia orgánica. Tesis de Maestría. Facultad de Ingeniería. Universidad del Valle. Cali, Colombia, 136 pp.

Bermúdez R.C., Rodríguez S., Martínez M.C. y Terry A.I. (2003). Ventajas del empleo de reactores UASB en el tratamiento de residuales líquidos para la obtención de biogás. RTQ XXIII, 37-44.

Bolle W.L., van Breugel J., van Eybergen G.C., Kossen, N.W. y Zoetemeyer R.J. (1986). Modeling the liquid flow in up-flow anaerobic sludge blanket reactors. Biotechnol. Bioeng. XVIII, 1615-1620.

Bracho N.R. (2003). Optimisation of faecal coliform removal performance in three tertiary maturation ponds. Tesis de Doctorado. Facultad de Ingeniería y Ciencias físicas. Universidad de Surrey, Inglaterra, $273 \mathrm{pp}$.

de Carvalho K.Q. (2006). Reposta dinâmica de reator UASB em escala piloto submetido a cargas orgânicas e hidráulicas cíclicas: modelos matemáticos e resultados experimentais. Tesis de Doctorado. Facultad de Ingeniería. Universidad de São Carlo. São Carlo, Brasil, 193 pp.

de Carvalho K.Q., Salgado M., Passig F y Pires E. (2008). Resposta dinâmica de reator UASB submetido a cargas orgânicas e hidráulicas cíclicas: resultados experimentais. Memorias. IX Taller y Simposio latinoamericano de Digestión Anaerobia. Valparaiso, Chile. 1 al 23 de octubre, pp. 513-519. CD-ROM.

Devore J.L. (2001). Probabilidad y estadística para ingeniería y ciencias. 5a ed. Thomson, Ciudad de México, Mexico, $762 \mathrm{pp}$.

Elgeti K. (1996). A new equation for correlating a pipe flow a reactor with a cascade of mixed reactors. Chem. Eng. Sci. 51, 5077-5080. DOI: 10.1016/S0009-2509(96)00342-9.

Gallegos M., Celis L.B. y Razo E. (2010). Competencia por sustrato durante el desarrollo de biomasa sulfatorreductora a partir de un lodo metanogénico en un reactor UASB. Rev. Int. Contam. Ambie. 26, 109-117.
Giácoman G., Frías J., Hurtado F.A., Coronado V. y Méndez R. (2003). Análisis de la hidrodinámica de un reactor de flujo pistón. Ingeniería 7, 7-19.

Gimenez J.R., Nassr S.C., Maestri R.D. y Monteggia, L.O. (2002). Physical modelling of an upflow anaerobic sludge blanket reactor: near-field study. Water Sci. Technol. 45, 157-162.

Grobicki A. y Struckey, D.C. (1992). Hydrodynamic characteristics of the anaerobic baffled reactor. Water Res. 26, 371-378.

DOI: 10.1016/0043-1354(92)90034-2.

Heertjes P.M. y Van der Meer R.R. (1978). Dynamics of liquid flow in an up-flow reactor used for anaerobic treatment of wastewater. Biotechnol. Bioeng. 20, 15771594. DOI: 10.1002/bit.260201007.

Iliuta I., Thyrion F.C. y Muntean O. (1998). Axial dispersión on liquid in gas-liquid concurrent downflow and upflow fixed-bed reactors with porous particles. Trans IChemE. 76, 64-72. DOI: $10.1205 / 026387698524488$.

Ji Y.X., Xing B.S., Yang, G.F., Ni W.M., Guo L.X. y Jin R.C. (2014). Performance and hydrodynamic features of a staged up-flow ANAMMOX sludge bed (SUASB) reactor. Chem. Eng. J. 253, 298-304.

DOI: $10.1016 /$ j.cej.2014.05.048.

Kim S.D. y Kim C.H. (1983). Axial dispersion characteristics of three phase fluidized beds. J. Chem. Eng. Jpn. 16, 172-177.

Kreft A. y Zuber A. (1978). On the physical meaning of the dispersion equation and its solutions for different initial and boundary conditions. Chem. Eng. Sci. 33, 1471-1480. DOI: 10.1016/0009-2509(78)85196-3.

Lara C., Díaz J. y Bona A. (2000). Evaluación hidráulica de un reactor UASB de compartimientos. Memorias. Seminario Internacional: Tratamiento y Postratamiento de Aguas Residuales. Tunja, Colombia. 12 al 13 de octubre, pp. 255-273.

Levenspiel O. (1999). Chemical reaction engineering. 2a ed. John Wiley and Sons Nueva York, EUA, 634 pp.

Lou S.J., Tartakovsk B., Zeng Y., Wu P. y Guiot, S.R. (2006). Fluorescence-based monitoring of tracer and substrate distribution in an UASB reactor. Chemosphere 65, 1212-1220.

DOI: 10.1016/j.chemosphere.2006.03.045.

Martin A.D. (2000). Interpretation of residence time distribution data. Chem. Eng. Sci. 55, 5907-5917.

DOI: 10.1016/S0009-2509(00)00108-1.

Morel E., Santamaria K., Perrier M., Guiot S.R. y Tartakovsky B. (2004). Application of multi-wavelength fluorometry for on-line monitoring of an anaerobic digestion process. Water Res. 38, 3287-3296. DOI: 10.1016/j.watres.2004.05.003. 
Morgan J.M., Jiménez B. y Noyola A. (1997). Tracer studies in laboratory and pilot scale UASB reactor. Environ. Technol. 18, 817-825.

Narnoli S.K. y Mehrotra I. (1997). Sludge blanket of UASB reactor: mathematical simulation. Water Res. 31, 715-726. DOI: 10.1016/S0043-1354(97)80987-6.

Peña M.R., Mara D.D. y Avella G.P. (2006). Dispersion and treatment performance analysis of an UASB reactor under different hydraulic loading rates. Water Res. 40, 445-452. DOI: 10.1016/j.watres.2005.11.021.

Pérez A. y Torres P. (2008). Evaluación del comportamiento hidrodinámico como herramienta para optimización de reactores anaerobios de crecimiento en medio fijo. Rev. Fac. Ing. Univ. Antioquia. 45, 27-40.

Pérez J.I. (2010). Modelo de dispersión axial de un reactor anaerobio de flujo ascendente (RAFA) utilizando aguas residuales municipales. Tesis de Doctorado. Facultad de Ingeniería. Universidad del Zulia, Maracaibo, Venezuela, $197 \mathrm{pp}$.

Pérez J.I. y Aldana G.J. (2013). Modelación física de un reactor anaerobio de flujo ascendente (RAFA). Rev. Técn. Ing. Univ. Zulia. 36, 153-163.

Pérez J.I., Aldana G.J. y Arguello G.J (2015). Dispersión de un reactor anaerobio de flujo ascendente (RAFA) usando un modelo matemático ajustado a las condiciones de borde. Memorias. $12^{\circ}$ Congreso Interamericano de Computación Aplicado a la Industria de Procesos, (CAIP). Cartagena, Colombia. 14 al 17 de septiembre, pp. 599-603. CD-ROM.
Ren T.T., Mu Y., Yu H.Q., Harada H. y Li Y.Y. (2008). Dispersion analysis of an acidogenic UASB reactor. Chem Eng J. 142, 182-189. DOI: 10.1016/j.cej.2007.11.028.

Rincón N., Galindo A. y Pérez J. (2011). Evaluación del comportamiento hidráulico en un reactor anaerobio de doble cámara (RADCA). Rev. Fac. Ing. Univ. Antioquia. 61, 53-63.

Singh K.S., Viraraghavan T. y Bhattacharyya D. (2006). Sludge blanket height and flow pattern in UASB reactors: Temperature effects. J. Environ. Eng. 132, 895-900.

DOI: 10.1061/(ASCE)0733-9372(2006)132:8(895).

Singhal A., James G., Praveen V.V. y Ramachandran K.B. (1998). Axial dispersion model for upflow anaerobic sludge blanket reactors. Biotechnol. Prog. 14, 645-648.

Van Der Meer R.R. y Herertjes P.M. (1983). Mathematical description of anaerobic treatment of wastewater in upflow reactors. Biotechnol. Bioeng. XXV, 2531-2556.

Wu B.M. y Hickey R.F. (1997). Dynamic model for UASB reactor including reactor hydraulics, reaction and diffusion. J. Environ. Eng. 123, 244-252.

Young H.W. y Young J.C. (1988). Hydraulic characteristics of upflow anaerobic filters. J. Environ. Eng. 114, 621-638.

DOI: 10.1061/(ASCE)0733-9372(1988)114:3(621).

Zeng Y., Mu S.J., Lou S.J., Tartakovsky B., Guiot S.R. y $\mathrm{Wu}$ P. (2005). Hydraulic modeling and axial dispersion analysis of UASB reactor. Chem. Eng. J. 25, 113-123. DOI: 10.1016/j.bej.2005.04.024. 